\title{
Distribution of the Inflammatory Lesions in the Central Nervous System of Dogs Affected with Disseminated and Ocular Form of Granulomatous Meningoencephalomyelitis
}

\author{
Tomomi MAEHARA ${ }^{1)}$, Akinori SHIMADA ${ }^{1) *}$, Takehito MORITA ${ }^{1)}$, Yuko SAWASHIMA ${ }^{1)}$ and Kou SAWASHIMA ${ }^{1)}$ \\ ${ }^{1)}$ Department of Veterinary Pathology, Tottori University, 4-101 Minami Koyama-cho, Tottori-shi, 680-8553, Japan
}

(Received 5 August 2008/Accepted 18 November 2008)

ABSTRACT. An aged Maltese dog (dog 1) showed gait ataxia for a month and suddenly died after convulsion. An aged Toy Poodle dog ( $\operatorname{dog} 2$ ) showed sudden blindness with unresponsive pupillary light reflexes and sudden death due to acute cardiac failure. Histological examination of the two dogs demonstrated severe granulomatous perivascular inflammation in the white matter throughout the central nervous system (CNS) including the brain and spinal cord. Based on the clinical and pathological findings, these dogs were diagnosed as granulomatous meningoencephalomyelitis (GME). In dog 2, the inflammatory changes predominated in the visual system such as the optic nerve, optic tract and optic radiation in the cerebrum (ocular form). Distribution pattern of the inflammatory lesions in the CNS was compared between the two dogs.

KEY WORDS: canine, granulomatous meningoencephalomyelitis, ocular form, optic neuritis.

J. Vet. Med. Sci. 71(4): 509-512, 2009

Granulomatous meningoencephalomyelitis (GME) is an acute, progressive inflammatory disease of the central nervous system (CNS) of dogs. Based on the clinical and pathological characteristics, GME has been classified to three forms: focal, disseminated and ocular forms [2, 3, 7]. The disseminated form of GME manifests acute onset of clinical signs suggestive of multifocal CNS disorder [7]. The ocular form of GME which can subsequently develop CNS involvements [7], is clinically characterized by acute onset of blindness and unresponsive to light stimulation, as a result of unilaterally or bilaterally optic neuritis occasionally with uveitis and retinal hemorrhage or detachment [2]. Pathological lesions of a disseminated form are usually distributed widely throughout the CNS [1, 3]. An ocular form occurs with granulomatous cuffs initially involving the optic nerves, optic disc, or retina, resulting in lesions in the retinal or postretinal portions of the optic nerves [9].

The ocular form has been reported less commonly than the other two forms; this may reflect selection bias rather than an absolute prevalence [7]. Mortality associated with the ocular form is low, and consequently, mild cases of ocular form that did not result in blindness may not have been recognized as ocular form. This case report describes detailed pathological changes of the ocular form of GME with the emphasis on the dominant distribution pattern of the inflammatory lesions in the visual system and its possible pathomorphogenesis by comparison of the findings in the disseminated form of GME. Clinical significance of GME in the differential diagnosis of the disease with visual disturbance is discussed elsewhere [8].

A 12 years old, female Maltese $\operatorname{dog}(\operatorname{dog} 1)$ showed gait

\footnotetext{
* Correspondence to: Shimada, A., Department of Veterinary Pathology, Tottori University, 4-101 Minami Koyama-cho, Tottori-shi, 680-8553, Japan.

e-mail: aki@muses.tottori-u.ac.jp
}

ataxia for a month and suddenly died after convulsion. An 11 years and 4 months old castrated Toy Poodle dog (dog 2) showed sudden blindness with unresponsive pupillary light reflexes; there were no abnormal findings in the electrical retinogram, suggesting presence of the responsible lesion in the visual system except for the retina. The dog 2 died due to acute cardiac failure resulting from rupture of chordae tendineae 2 months after the onset of the blindness.

After complete necropsy, samples from a variety of organs including the right eyeball, right optic nerve, brain, heart and lung were collected and fixed in 10\% neutral buffered formalin. Paraffin sections of $3 \mu \mathrm{m}$-thick were cut and stained with hematoxylin and eosin (HE). Lectin histochemistry with the use of biotinylated lectin Ricinus communics agglutinin I (RCA-1: specific marker for microglia/ macrophage, dilution 1:400) (Vector Laboratory, Burlingame, U.S.A.) [10] was performed on the selected sections from the brain and optic nerve. Immunohistochemistry with the use of monoclonal antibody to glial fibrillary acid protein (GFAP, dilution 1:500) (DakoCytomation, Glostrup, Denmark) was also performed on sections from the retina.

On gross examination, dilatation of both sides of the lateral ventricles was observed in the dog 1 . Histopathology demonstrated perivascular granulomatous inflammatory lesions in the white matter throughout the CNS including the cerebrum, cerebellum and spinal cord. The granulomatous lesions consisted of lymphocytes, plasma cells, epithelioid cells and occasional neutrophils. Severe inflammatory lesions were observed in the brain stem, cerebellum and spinal cord; the eyeball and optic nerve were not affected (Fig. 1). Combined with pathological and clinical findings, this case was diagnosed as disseminated form of GME.

There were no significant gross changes in all organs examined in the dog 2. Histopathology demonstrated 


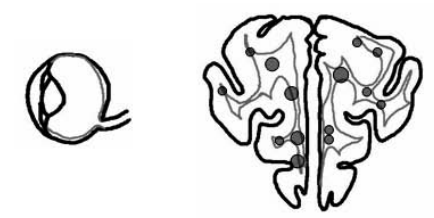

Optic nerve

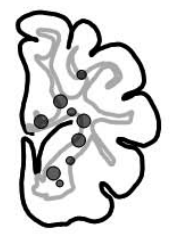

Striatum

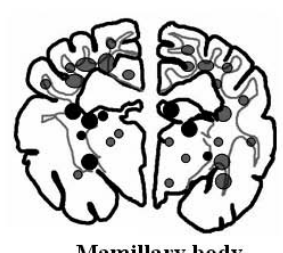

Mamillary body

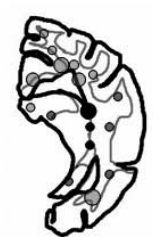

Hippocampu

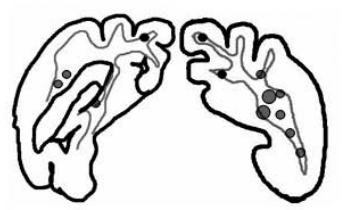

Occipital lobe

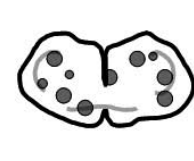

Corpus trapezoideum

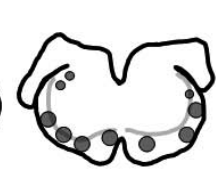

Medulla oblongata

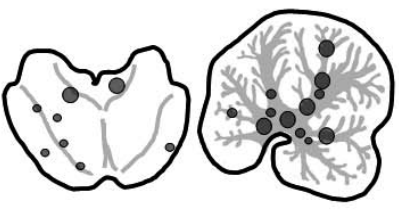

Pons

Cerebellum (vermis)

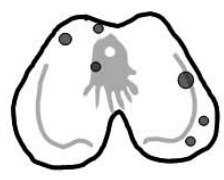

Mesocephalon

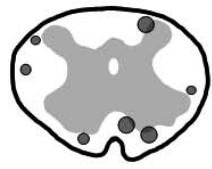

Spinal cord C8

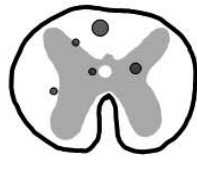

Spinal Cord T5

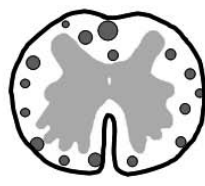

Spinal Cord L4
ㅇ : mild

: severe

: visual system

Fig. 1. Schematic diagram of the distribution of the granulomatous lesions in the central nervous system (CNS) in the dog 1, showing wide spread of the lesions throughout the CNS.
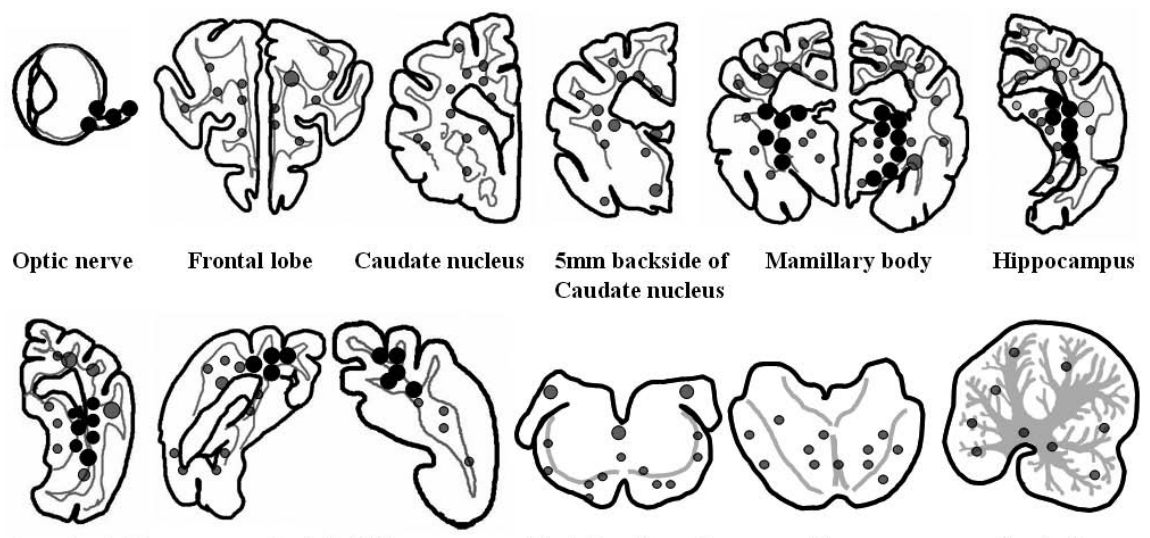

$5 \mathrm{~mm}$ backside of Hippocampus

Occipital lobe

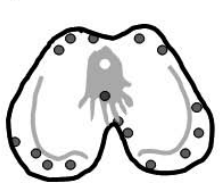

Mesocephalon

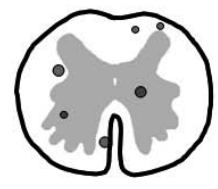

Spinal cord $\mathrm{C} 7$

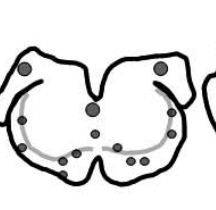

Medulla oblongata

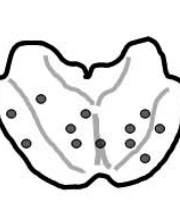

Pons

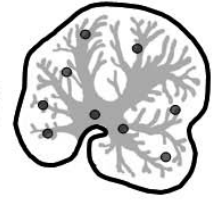

Cerebellum (vermis)

Fig. 2. Schematic diagram of the distribution of the granulomatous lesions in the CNS in the dog 2 . Note that lesions are predominated in the visual system, including the optic nerve, optic tract, corpus geniculatum laterale, radiatio optica and subcortical white matter at the visual cortex (occipital lobe).

perivascular granulomatous inflammatory lesions throughout the white matter in the CNS. The granulomatous lesions predominated in the visual system (Fig. 2), such as the optic nerve (Fig. 3), optic tract (Fig. 4), corpus geniculatum later- ale, radiatio optica and subcortical white matter at the visual cortex (occipital lobe); lesions in the optic nerve were most severe. Similar inflammatory changes were also observed in the acoustic nerve. The granulomatous lesions consisted 


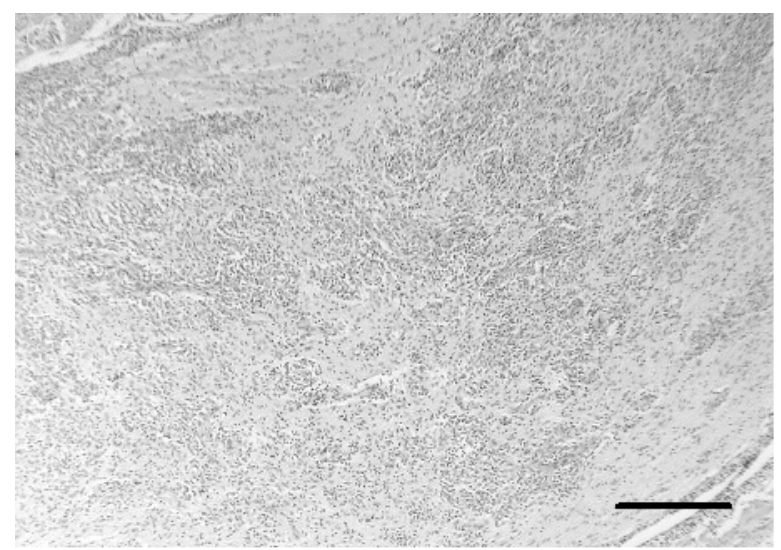

Fig. 3. Severe granulomatous lesions are shown in the optic nerve. Dog 2. H.E. Bar, $300 \mu \mathrm{m}$.

of same inflammatory cells as observed in dog 1; epithelioid cells in the lesions were positive for lectin RCA-1 (Fig. 5). Thinning of the optic nerve layer and marked loss of ganglion cells with mild to moderate astrogliosis, which was demonstrated by GFAP immunohistochemistry, were observed in the retina. No changes were seen in the other layers of retina.

In the dog 2, granulomatous inflammatory changes predominated in the visual system; a lesion in the optic nerve was most severe, suggesting optic neuritis as a primary lesion. An ocular form of GME with severe granulomatous optic neuritis was also reported [4, 10]. The dog 2 showed no nervous signs except for blindness. Combined with pathological and clinical findings, this case was diagnosed as ocular form of GME. Marked losses of ganglion cells with mild to moderate astrogliosis were observed exclusively in the optic nerve fiber layer and ganglion cell layer in the retina. In contrast, no changes were seen in the other layers of retina. These findings suggest that ganglion cell loss may be induced by the optic nerve loss resulting from severe optic neuritis. Clinical significance of the dominant lesions in the retina and optic nerve in the dog 2 is discussed elsewhere [8].

The disseminated form of GME is characterized by acute onset of neurological signs suggestive of multifocal CNS disorder [7]. The dog 1, diagnosed as disseminated form of GME, showed acute clinical signs with multifocal lesions throughout the CNS. On the other hand, the ocular form of GME is clinically characterized by acute onset of blindness associated with initial involvement of the lesions in the visual system [9]. Distribution patterns of the histological lesions observed in the present dogs corresponded to the neurological findings shown in each animal.

The detailed cause of GME is unknown [6]. GME might be $\mathrm{T}$ cell-mediated delayed-type hypersensitivity of an organ-specific autoimmune disease; the distribution pattern of the lesions involving central white matters and optic nerve suggests association of certain antigen in the develop-

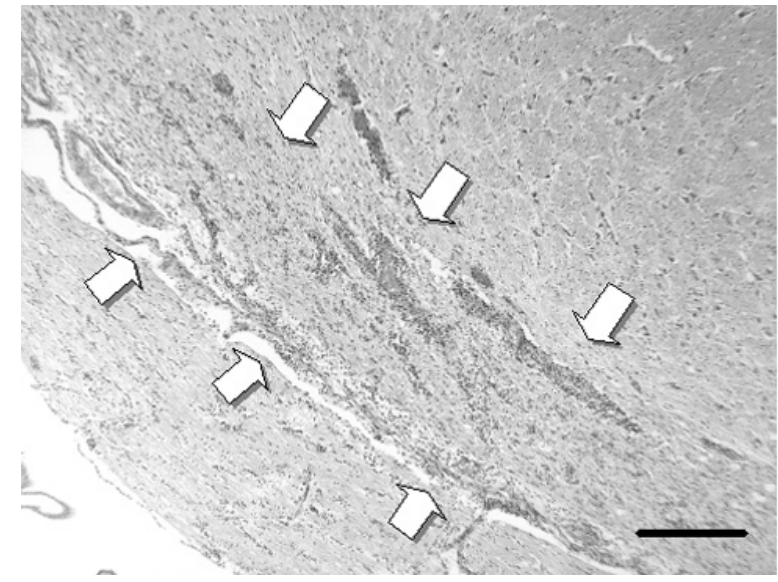

Fig. 4. Transverse section of the cerebrum at mamillary body. Granulomatous lesions are exclusively observed in the optic tract (arrows). Dog 2. H.E. Bar, $300 \mu \mathrm{m}$.

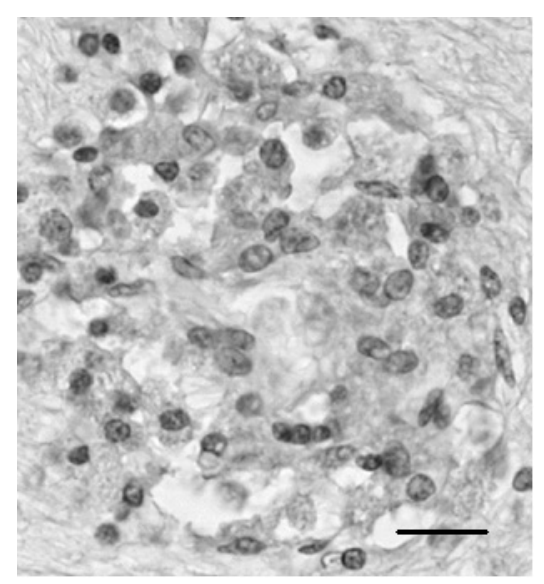

Fig. 5. A granulomatous lesions in the hippocampus. Epithelioid cells show positivity for lectin RCA-1. Dog 2. Bar, $10 \mu \mathrm{m}$.

ment of the disease [5]. In the dog 2, granulomatous inflammatory changes predominated in the white matter of the visual system, from the optic pupilla to subcortical white matter at the visual cortex (occipital lobe). Granulomatous inflammatory changes were also observed in the acoustic nerve in the $\operatorname{dog} 2$. Possible selective involvement of the nerve fibers and sensory system in the initial development of the disease and its significance in the pathogenesis would be a matter of further study.

\section{REFERENCES}

1. Braund, K. G. 1985. Granulomatous meningoencephalomyelitis. J. Am. Vet. Med. Assoc. 186: 138-141.

2. Collins, B. K. and Moore, C. P. 1991.Canine anterior Uvea. pp. 253-294. In: Veterinary Ophthalmology. 2nd ed. (Gelatt, K. N. ed.), Lee \& Febiger. Philadelphia.

3. de Lahunta, A. 1983. Small animal neurologic examination and 
index of disease of the nervous system. pp. 365-387. In: Veterinary Neuroanatomy and Clinical Neurology. 2nd ed. (Lahunta, De. ed.), W.B. Saunders Company, Philadelphia.

4. Garmer, N. L., Naeser, P. and Bergman, A. J. 1981. Reticulosis of the eyes and the central nervous system in a dog. J. Small. Anim. Pract. 22: 39-45.

5. Kipar, A., Baumgärtner, W., Vogl, C., Gaedke, K. and Wellman, M. 1998. Immunohistochemical characterization of inflammatory cells in brains of dogs with granulomatous meningoencephalomyelitis. Vet. Pathol. 35: 43-52.

6. Maxie, M. G. and Youssef, S. 2007. Nervous system. pp. 281458. In: Pathology of Domestic Animals. vol. 1, 5th ed. (Maxie, M.G. ed.), Elsevier, Philadelphia.

7. Munana, K. R. and Luttegen, P. J. 1998. Prognostic factors for dogs with granulomatous meningoencephalomyelitis: 42 cases
(1982-1996). J. Am. Vet. Med. Assoc. 212: 1902-1906.

8. Sawashima, Y., Sawashima, K., Shitaka, H., Morita, T., Maehara, T. and Shimada, A. 2008. A case of ocular form of granulomatous meningoencephalomyelitis in a dog. J. Jap. Vet. Med. Assoc. 61: 800-803 (in Japanese).

9. Summers, B. A., Cummings, J. F. and de Lahunta, A. 1995. Inflammatory disease of the central nervous system. pp. 95188. In: Veterinary Neuropathology. (Summers, B. A., Cummings, J. F. and de Lahunta, A. eds.) Mosby-Year Mook, St.Louis.

10. Suzuki, M., Uchida, K., Morozumi, M., Yanai, T., Nakayama, H., Yamaguchi, R. and Tateyama, S. 2003. Comparative pathological study on granulomatous meningoencephalomyelitis and central malignant histiocytosis in dogs. J. Vet. Med. Sci. 65: 1319-1324. 